\title{
Design of segmented off-diagonal thermoelectric generators using topology
} optimization

\author{
Lundgaard, Christian; Sigmund, Ole
}

Published in:

Applied Energy

Link to article, DOI:

10.1016/j.apenergy.2018.12.021

Publication date:

2019

Document Version

Peer reviewed version

Link back to DTU Orbit

\section{Citation (APA):}

Lundgaard, C., \& Sigmund, O. (2019). Design of segmented off-diagonal thermoelectric generators using topology optimization. Applied Energy, 236, 950-960. https://doi.org/10.1016/j.apenergy.2018.12.021

\section{General rights}

Copyright and moral rights for the publications made accessible in the public portal are retained by the authors and/or other copyright owners and it is a condition of accessing publications that users recognise and abide by the legal requirements associated with these rights.

- Users may download and print one copy of any publication from the public portal for the purpose of private study or research.

- You may not further distribute the material or use it for any profit-making activity or commercial gain

- You may freely distribute the URL identifying the publication in the public portal

If you believe that this document breaches copyright please contact us providing details, and we will remove access to the work immediately and investigate your claim. 


\title{
Design of segmented off-diagonal thermoelectric generators using topology optimization
}

\author{
Christian Lundgaard, Ole Sigmund
}

Department of Mechanical Engineering Techinical University of Denmark, Nils Koppels Allé 404, DK-2800 Kgs. Lyngby, Denmark

\begin{abstract}
A density-based topology optimization methodology is used to optimize the off-diagonal figure-of-merit and off-diagonal electrical power output of thermoelectric generators by distributing two different thermoelectric active materials in a two dimensional design space. Off-diagonal thermoelectric generators are characterized by converting a vertical thermal heat flux into a horizontal electric current, and may be useful in applications where the electrodes connected to the generator are prone to thermo-mechanical stress and wear.

With basis in the topology optimization framework and a sequence of numerical examples, we discuss critical and important model parameters such as objective functions, heat transfer rates and device lengths. All results are supported by exhaustive crosschecks and validations, and it is shown that the off-diagonal figure-of-merit and the off-diagonal electrical power output may be improved by $233 \%$ and $229 \%$, respectively, compared to other optimization approaches available in the literature.
\end{abstract}

Keywords: Topology optimization, thermoelectric energy conversion, figure-of-merit, electric power output, thermoelectricity, renewable energy

\section{Introduction}

Thermoelectric generators exploit the Seebeck effect to convert thermal energy into electric energy [1]. This type of device is especially useful in waste heat recovery applications as $55-75 \%$ of the fuel consumption in conventional combustion engines is converted into waste heat [2]. By using thermolectric generators in waste heat recovery applications, it is possible to increase the overall efficiency which may be profitable even for small performance improvements. With a point of departure in thermoelectric generators, we present and solve a numerical design problem which aims at increasing the performance of a special type of thermoelectric generators called off-diagonal thermoelectric generators.

With reference to the sketch in Fig. 1, off-diagonal thermoelectric generators convert a vertical heat flux into a horizontal electrical current. As the electrodes are disconnected from the thermal reservoirs, the thermomechanical stress and wear of the electrodes are reduced, which makes off-diagonal thermoelectric generators suitable in e.g. applications where the temperature difference between the hot and the cold reservoirs varies considerably during operation.

Preprint submitted to Applied Energy
The present study is inspired by the work of Sakai et al. [3] and takes point of departure in an optimization problem of an off-diagonal thermoelectric generator serving in a realistic application. Sakai et al. [3] demonstrated that it is possible to increase the off-diagonal figure-ofmerit and electrical power output for this type of device, by determining the optimal tilting angle and volume fraction between two thermoelectric active materials placed in a layered configuration.

As we point our attention to the layered configuration of Sakai and coworkers' design solutions, we presume that the performance of the design solutions can be increased further by allowing geometrically free distribution of the two materials. Such a design problem can be powered by a numerical optimization approach called topology optimization $[4,5]$ which we use in the present study.

With basis in the schematic of the design problem in Fig. 1, the goal of the topology optimization problem is to increase the figure-of-merit and electrical power output of the off-diagonal thermoelectric generators by distributing two different thermoelectric materials, Material $\mathrm{A}$ and Material $\mathrm{B}$, in the two dimensional design space, $\Omega_{D}$. The material parameters of Material A and B 
have been listed in Tab. 1.

The work of Sakai et al. [3] is particularly interesting for our endeavor, as the theoretically obtained design solutions were both manufactured and experimentally verified. Despite the neglection of parasitic losses between the layers of different types of materials, Sakai and coworkers found excellent agreement between the analytical predictions and experimental testing. Due to these observations, we find it reasonable to assume that Sakai and coworkers overcame challenges concerning manufacturability and minimization of parasitic losses. This topic is further discussed in Secs. 3.1 and 3.2.

The topology optimization methodology used in the present study is powered by the finite element method $[7,8]$, adjoint sensitivity analysis [9] and the method of moving assymptotes [10]. The finite element method is a discretization approach used to estimate the solution to the governing partial differential equations. Adjoint sensitivity analysis provides the gradients of the objective function with respect to the design variable field, which are used in the deterministic gradient-based optimization solver called the method of moving assymptotes. The method of moving assymtotes is an approach used to solve the design problem and ensure convergence of the design routine within a couple of hundred design iterations despite solving problems with more than 10,000 design variables. Interested readers are referred to Lundgaard and Sigmund [6] who provide a detailed introduction to the methodology.

The topology optimization approach is related to wellaccepted studies in the thermoelectric literature such as functionally graded materials $[1,11]$, the compatibility approach [12], genetic sizing approaches [13] and homogenization $[3,14,15,16]$, however the methodology takes a completely different off-set and modeling approach, and converges to very different design solutions and hereby opens a hole new branch of optimization approaches for off-diagonal thermoelectric generators.

\subsection{Physical model}

The topology optimization framework is based on the temperature independent and steady state form of Fourier's and Ohm's generalized equations which are given by $[7,1]$ :

$$
\begin{aligned}
& \frac{\partial Q_{i}}{\partial x_{i}}=\dot{q} \text { in } \Omega_{D} \\
& \frac{\partial J_{i}}{\partial x_{i}}=0 \text { in } \Omega_{D} \\
& Q_{i}=T \alpha_{i j} J_{j}-\kappa_{i j} \frac{\partial T}{\partial x_{j}}
\end{aligned}
$$

Electrical

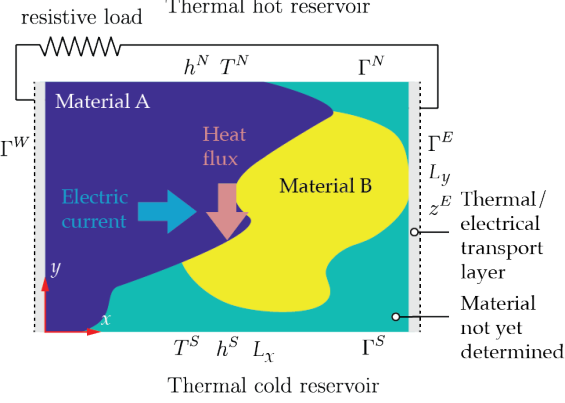

Figure 1: A sketch of the off-diagonal thermoelectric generator design problem. The temperature difference between the hot exhaust gas from e.g. a combustion engine and the cold ambient air generates a heat flux from $\Gamma^{N}$ to $\Gamma^{S}$. The heat flux is converted into a vertical electrical current by exploiting the Seebeck effect. By spatially distributing Material A and B (see Tab. 1) in the two dimensional design space, $\Omega_{D}$, the off-diagonal figure-of-merit or electrical power output are optimized. The thermal heat transfer between the thermal hot and cold reservoirs and the thermoelectric generator are modeled by Newton's law of cooling, see Sec. 1.3.

$$
J_{i}=\sigma_{i j}\left(E_{j}-\alpha_{i k} \frac{\partial T}{\partial x_{k}}\right)
$$

where $Q_{i}$ is the heat flow density; $x_{i}$ is the spatial coordinates; $\dot{q}=J_{i} E_{i}$ is the Joule heating term; $T$ is the temperature; $\alpha_{i j}$ is the Seebeck coefficient; $J_{i}$ is the electric current density; $\kappa_{i j}$ is the thermal conductivity of the medium; $\sigma_{i j}$ is the electrical conductivity of the medium; and $E_{j}=-\partial V / \partial x_{j}$ is the electric field. The tensor indices, $i$ and $j$, have two entries, $x$ and $y$, which are corresponding to the spatial directions in a Cartesian coordinate system.

\subsection{External resistive load}

The electric current in the external resistive load is given by:

$$
n_{i} J_{i}=z^{\square}\left(V-V^{\square}\right)
$$

where $\square$ indicates the surface on which the boundary condition is imposed (north, south, east, west, see Fig 1 for definitions), $z^{\square}$ is the impedance of the external resistive load, $n_{i}$ is the unit vector normal and $V^{\square}$ is a reference electric potential.

\subsection{Newton's law of cooling}

With reference to the simple rectangular design and the relatively small length scales of the thermoelectric generator problem sketches in Fig. 1, we assume that the 
Table 1: The Seebeck coefficient, $\alpha$; the electric conductivity, $\sigma$; and the thermal conductivity, $\kappa$, for Material A and B used in the design problems. The material parameters are similar to the material parameters used in [3].

\begin{tabular}{lrrrrr}
\hline & Color in plots & $\alpha[\mathrm{V} / \mathrm{K}]$ & $\sigma[\mathrm{S} / \mathrm{m}]$ & $\kappa[\mathrm{W} /(\mathrm{m} \cdot \mathrm{K})]$ & $Z[1 / \mathrm{K}]$ \\
\hline Material A & & $210 \cdot 10^{-6}$ & $8.333 \cdot 10^{4}$ & 1.1 & $3.3409 \cdot 10^{-3}$ \\
Material B & $-20 \cdot 10^{-6}$ & $588.2 \cdot 10^{4}$ & 51.0 & $0.0461 \cdot 10^{-3}$ \\
\hline
\end{tabular}

Table 2: List of important variables used throughout the paper.

\begin{tabular}{ll}
\hline Variable & Description \\
\hline$\Gamma^{N}$ & Northern boundary (cold reservoir) \\
$\Gamma^{S}$ & Southern boundary (hot reservoir) \\
$\Gamma^{E}$ & Eastern boundary \\
$\Gamma^{W}$ & Western boundary (electrically grounded) \\
\hline$T^{N}$ & Temperature at $\Gamma^{N}$ (cold reservoir) \\
$T^{S}$ & Temperature at $\Gamma^{S}$ (hot reservoir) \\
$T^{N S}$ & Abbreviation of $T^{N}$ and $T^{S}$ combined \\
\hline$h^{N}$ & Convection coefficient at $\Gamma^{N}$ \\
$h^{S}$ & Convection coefficient at $\Gamma^{S}$ \\
$h^{N S}$ & Abbreviation of $h^{H}$ and $h^{C}$ combined \\
\hline$\vec{T}$ & The temperature field [K] \\
$\vec{V}$ & The electric potential field [V] \\
$\vec{Q}_{x}, \vec{Q}_{y}$ & The thermal heat flux field \\
$\vec{J}_{x}, \vec{J}_{y}$ & in $x$ and $y$, respectively \\
& The electric current density \\
\hline$z^{E}$ & field in $x$ and $y$, respectively \\
& Electrical resistance in \\
\hline$f_{Z}$ & outer electrical load at $\Gamma^{E}$ \\
$f_{P}$ & Off-diagonal figure-of-merit \\
$f_{P} / L_{x}$ & Off-diagonal electrical power output \\
\hline$\Omega_{D}$ & Normalized off-diagonal \\
$L_{x}$ & Lectrical power output \\
$L_{y}$ & Length of $\Omega_{D}$ in $x$ \\
\hline & \\
\hline
\end{tabular}

heat transfer between the hot and the cold reservoirs and the thermoelectric generator is governed by Newtons law of cooling.

By modeling heat transfer with Newtons law of cooling, it is assumed that the thermal heat transfer between thermal hot and cold reservoirs and the module is proportional to the temperature differences between these. Mathematically, Newton's law of cooling is given by:

$$
n_{i} q_{i}=h^{\square}\left(T-T^{\square}\right)
$$

where $q_{i}$ is the heat flux due to convection, $h^{\square}$ is the convection coefficient and $T^{\square}$ is the temperature of the reservoir.

\subsection{Finite element formulation}

To set up the topology optimization framework, we introduce a design field, $\rho$, such that Eqs. (1) become functions of $\rho$, i.e. $\alpha=\alpha(\rho), \sigma=\sigma(\rho)$ and $\kappa=\kappa(\rho)$. The discretized finite element equations suited for the topology optimization framework can now be obtained by multiplying the strong forms of Eqs. (1) with suitable test functions; integrating over the domain; performing integration by parts of higher dimensions on relevant terms and introducing the design field dependent interpolation functions $[7,17,8]$.

The design variable field makes it possible to compute the gradients of the objective functions with an analytic adjoint sensitivity analysis. The gradients of the objec tive function contain the information which describe how Material A and Material B should be spatially distributed to maximize the objective functions.

A detailed description of the derivation and implementation of the optimization framework is provided [6].

\subsection{The design problem}

To provide the best comparison between the optimization approaches, we decided to take basis in the same pair of objective functions as the main references of the present study, the works of Yang et al. [14] and Sakai et al. [3]. The objective functions are the off-diagonal figure-of-merit and the off-diagonal electrical power output. 
Table 3: The boundary conditions used to evaluate the figure-of-merit, $f_{Z}$, in Eqs. (5). To compute the figure-of-merit, it is necessary to solve three different finite element problems with the boundary conditions listed below.

\begin{tabular}{lccccc}
\hline & & \multicolumn{4}{c}{ Boundary } \\
\cline { 2 - 6 } Load-case & & $\Gamma^{N}$ & $\Gamma^{S}$ & $\Gamma^{E}$ & $\Gamma^{W}$ \\
\hline$\alpha$ & $T$ & $10^{-5}$ & 0 & - & - \\
& $V$ & - & - & - & 0 \\
\hline \multirow{2}{*}{$\sigma$} & $T$ & - & - & 0 & 0 \\
& $V$ & - & - & $10^{-5}$ & 0 \\
\hline \multirow{2}{*}{$\kappa$} & $T$ & $10^{-5}$ & 0 & - & - \\
& $V$ & 0 & - & - & - \\
\hline
\end{tabular}

With reference to Fig. 1, we denote the northern, southern, eastern and western surfaces of the design domain $\Gamma^{N}, \Gamma^{S}, \Gamma^{E}, \Gamma^{W}$, respectively. The design domain is rectangular with length, $L_{x}$, and height, $L_{y}$, length-toheight ratio $\Lambda=L_{x} / L_{y}$; and an outer electric impedance, $z^{E}$, is imposed on boundary $\Gamma^{E}$. For readability purposes we have listed the most used abbreviations and variables used throughout the paper in Tab. 2. The model parameters are similar to the model parameters used in [3].

\subsubsection{Figure-of-merit}

The thermoelectric off-diagonal figure-of-merit, $f_{Z}$, is computed by the following composite objective function:

$$
f_{Z}=\frac{f_{\alpha}{ }^{2} f_{\sigma}}{f_{\kappa}}
$$

where $f_{\alpha}$ is the objective function for the Seebeck coefficient, $f_{\sigma}$ is the objective for the electric conductivity and $f_{k}$ is the objective for the thermal conductivity. In the following we will account for how to compute these objective functions.

The off-diagonal figure-of-merit is computed by solving three different finite element problems or load cases, computing the corresponding objective functions and multiplying them as stated in Eq. (4). The boundary conditions for the $\alpha$-load case, the $\sigma$-load case and the $\kappa$-load case have been listed in Tab. 3.

By computing the objective functions in Eq. (4) with small differences in the state fields as listed in Tab. 3, the governing equations remain in the linear regime where the Joule heating is negligible. By including the surface electrodes and ensuring that the governing equations stay in the linear regime, the evaluation of the objective functions are consistent with the analytic and experimental approaches seen in e.g. Rowe [1], Yang et al. [14, 18] and Sakai et al. [3].

The objective functions in Eq. (4) can now be computed by the following surface integrals, please cf. Fig. 1.

$$
\begin{aligned}
f_{\alpha}=\frac{L_{y}}{L_{x}} & {\left[\frac{1}{L_{y}} \int_{\Gamma^{E}} V \mathrm{~d} S-\frac{1}{L_{y}} \int_{\Gamma^{W}} V \mathrm{~d} S\right] . } \\
& {\left[\frac{1}{L_{x}} \int_{\Gamma^{N}} T \mathrm{~d} S-\frac{1}{L_{x}} \int_{\Gamma^{S}} T \mathrm{~d} S\right]^{-1} } \\
f_{\sigma}=\frac{L_{x}}{L_{y}} & {\left[\int_{\Gamma^{E}} J_{x} \mathrm{~d} S\right] . } \\
& {\left[\frac{1}{L_{y}} \int_{\Gamma^{E}} V \mathrm{~d} S-\frac{1}{L_{y}} \int_{\Gamma^{W}} V \mathrm{~d} S\right]^{-1} } \\
f_{\kappa}=\frac{L_{y}}{L_{x}} & {\left[\int_{\Gamma^{N}} Q_{y} \mathrm{~d} S\right] . } \\
& {\left[\frac{1}{L_{x}} \int_{\Gamma^{N}} T \mathrm{~d} S-\frac{1}{L_{x}} \int_{\Gamma^{S}} T \mathrm{~d} S\right]^{-1} }
\end{aligned}
$$

As a reference, the integral expressions above are often in one dimensional modeling written as $f_{\alpha}=\Delta V / \Delta T$, $f_{\sigma}=J / \Delta V$ and $f_{\kappa}=Q / \Delta T$, where $\Delta T$ and $\Delta V$ denote the temperature and electric potential difference between the boundaries, respectively.

The reporting of the intricated integral expressions in Eq. (5) is serving as guidance for future research, as the expressions explicitly state the approach for computing the objective functions in Eq. (4).

\subsubsection{The electrical power output}

The off-diagonal electric power output, $f_{P}$, is given by:

$$
f_{P}=\frac{1}{L_{y}} \int_{\Gamma^{E}} V \mathrm{~d} S \int_{\Gamma^{E}} J_{x} \mathrm{~d} S
$$

which is simply an integral expression, where the average electric potential along $\Gamma^{E}$ is multiplied with the integral of the electric current density along $\Gamma^{E}$. The corresponding objective function in one dimensional modeling is often written as $P=V J$, where $P$ is the electrical power output, $V$ is the electrical potential and $J$ is the electric current.

By assuming that a highly conductive surface electrode is connected to $\Gamma^{E}$, the boundary conditions can be written as listed in Tab. 4. The height of the design domain $\left(L_{y}=0.004[\mathrm{~m}]\right)$ and temperatures of the hot and cold reservoirs are equivalent to the design problems investigated in Sakai et al. [3]. 
Table 4: The boundary conditions used to evaluate the electrical power output, $f_{P}$, for off-diagonal thermoelectric generators.

\begin{tabular}{lcccc}
\hline & \multicolumn{4}{c}{ Boundary } \\
\cline { 2 - 5 } & $\Gamma^{N}$ & $\Gamma^{S}$ & $\Gamma^{E}$ & $\Gamma^{W}$ \\
\hline$T^{\square}[\mathrm{K}]$ & 85 & 0 & - & - \\
$h^{\square}[\mathrm{W} / \mathrm{m} \cdot \mathrm{K}]$ & $10^{4}$ & $10^{4}$ & - & - \\
$V^{\square}[\mathrm{V}]$ & - & - & - & 0 \\
$z^{\square}[\mathrm{S} / \mathrm{m}]$ & - & - & $z^{E}$ & - \\
\hline
\end{tabular}

\subsubsection{Sensitivities, filter operations and optimizer}

The gradients of the objective functions in Eqs. (5) and (6) with respect to the design variable field are computed by the discrete adjoint approach (see Bendsøe and Sigmund [5]). To ensure length-scale control and robustness toward manufacturing variations, the optimization problem is formulated in a min/max form for three different projected realizations of the design variable field [19]. The physical design variables used for the finite element analysis are obtained by a density filter and a Heaviside projection filter operation [20, 21] and the optimization problem is solved with the method of moving assymptotes [10]. Readers interested in implementation details of the framework are referred to Lundgaard and Sigmund [6].

\section{Results}

To demonstrate that the topology optimization framework is suited for optimizing the figure-of-merit and the electrical power output for off-diagonal thermoelectric generators, we present a sequence of different numerical examples throughout this section.

The framework is benchmarked against the analytical predictions and frameworks in Sec. 2.1, design problems solved for the figure-of-merit are presented in Sec. 2.2 and design problems solved for the electric power output are presented in Sec. 2.3. The performance of off-diagonal, diagonal thermoelectric generators are compared in Sec. 2.4 and the thermal heat transfer modeling with Newton's law of cooling is discussed in Sec. 2.5.

\subsection{Benchmark examples}

The topology optimization framework used in the present study is validated with basis in the analytic optimization approach presented in Sakai et al. [3]. The objective of the analytical approach is to increase the offdiagonal figure-of-merit, $f_{Z}$, and the off-diagonal electric power output, $f_{P}$, by determining the optimal tilting angle between two thermoelectric materials placed in a layered configuration.

The analytical approach by Sakai et al. [3] is derived under the assumption of infinite periodicity and negligible boundary effects. Design solutions of infinite periodicity are assumed to consist of layered, and infinitely narrow channels. Boundary effects refer to the disturbances in the state fields that occur in the transitions between the material phases and near the outer boundaries of design solutions.

As infinite periodic design solutions are a purely mathematical concept, we have to distinguish between design solutions with finitely and infinitely small design features. Equivalent to the design solutions manufactured and tested by Sakai et al. [3], our design solutions with finite length scales have an enforced minimum physical length-scale relative to the design domain height of 0.05 . The corresponding design solutions for infinitely small design features do not have such minimum length-scale.

With reference to Figs. 3a and 3b, layered designs with finite and infinite periodicity are here called finite layered designs and infinite layered designs, respectively. To validate the numerical framework, we have plotted the relationship between the figure-of-merit and the tilting angle for the different optimization approaches in Fig. 2.

The entries in Fig. 2 are computed with basis in the three different analytical design solutions in Fig. 3: (A) The infinite layered design without boundary effects in Fig. 3a, (B) the infinite layered design with boundary effects in Fig. 3b and (C) the finite layered design with boundary effects in Fig. 3b. The design solutions are all solved for $\Lambda=10, V=0.5$ and $\theta \in[0 ; 90]$ and the positions of the integration domains in Eq. (5) have been marked by black, green and blue encircled rectangles on the design solutions plots.

With reference to Fig. 2, the relationship between $f_{Z}$ and $\theta$ for the infinite layered designs without boundary effects and the analytical predictions in Sakai et al. [3] show excellent agreements, as the numerical simulations and the analytical derivations are carried out under the same assumptions: Infinite periodicity and negligible boundary effects. As the analytical and numerical optimization frameworks show excellent agreement, we confidently conclude that the topology optimization framework and underlying finite element model are suited for solving off-diagonal thermoelectric generator design problems seen in Fig. 1.

The influence of the boundary effects and the variations in the state fields over the layers cause the relationship between $f_{Z}$ and $\theta$ for the different designs to deviate. The differences between the numerical and analytical 
optimization framework can be minimized by narrowing the channels, increasing the length-to-height ratio, and placing the integration domains "sufficiently far" from the outer boundaries.

The physically realizable design solutions with finite layered design with boundary effects obtained with the approach presented in Sakai et al. [3] are in the remaining part of the paper simply denoted analytical design solutions.

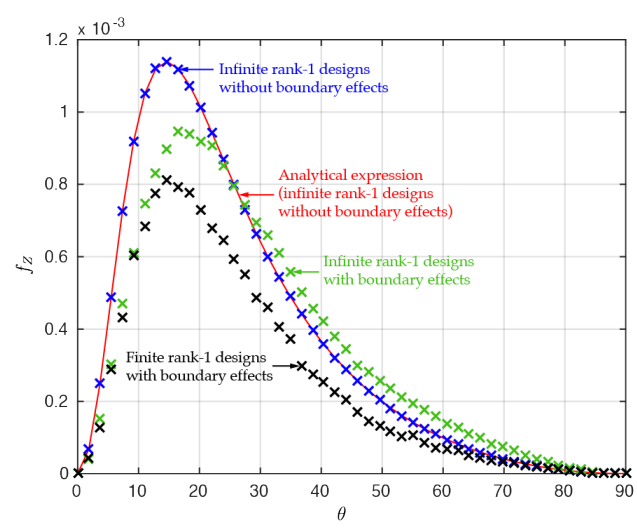

Figure 2: The figure-of-merit, $f_{Z}$, as function of the tilting angle, $\theta$, for the design solutions in Fig. 3. The numerical evaluation of the infinite layered designs without boundary effects have the same predicted performance as the analytical predictions and experimental results (not shown on the plot) presented in the work of Sakai et al. [3]. Due to the excellent fir predictions and the experimental measurement, we conclude that the framework used in the present study is suited for solving off-diagonal thermoelectric generator design problems.

\subsection{Figure-of-merit}

The goal of the first numerical design problem is to optimize the off-diagonal figure-of-merit, $f_{Z}$. To demonstrate what can be achieved in performance improvements with the proposed methodology, we have decided to use the design solutions obtained with the analytical approach as benchmarks for the numerical approach.

The length-to-height ratio of the design domain, $\Lambda$, is an important model parameter so we have solved 100 different design problems for $\Lambda \in[0.1 ; 10]$, and plotted a selection of the design solutions in Fig. 4. A selection of the corresponding design solutions solved with the analytical approach have been plotted in Fig. 5. The design solutions solved with the analytical optimization approach are obtained by a simple and computational expensive parameter study over $\theta$ which is swept in 100 different and equally spaced steps in the interval $\theta \in$ $[0 ; 90]$ for each value of $\Lambda$.

By comparing Fig. 4 and Fig. 5, we point out that the numerical and analytical approach converge to very different design solutions. The large difference is also reflected in performance of the design solutions which have been plotted in Fig. 6 .

The plot shows the relationship between $f_{Z}$ and $\Lambda$ for the design solutions solved for $f_{Z}$ and $f_{P}$ (see Sec. 2.3 for more information). The important message of the plot is the following: The design solutions solved for $f_{Z}$ with the numerical approach outperform the design solutions solved for other objectives and other optimization approaches with up to $233 \%$. With basis in this, we hereby conclude that the numerical optimization approach is suited for solving design problems of off-diagonal thermoelectric generators

\subsubsection{The features of the design solutions}

In this section we explain why there occurs an optimum between $f_{Z}$ and $\Lambda$ in Fig. 6 and how we can use this knowledge to increase the performance of thermoelectric generators. With reference to the figure, we start this discussion by pointing out that the largest $f_{Z}$ is achieved for $\Lambda \approx 1.3$.

As seen in the state field plots for the design solutions in Fig. 7, this specific length-to-height ratio provides the ideal interplay between a high electric conductivity in the horizontal direction, a low thermal conductivity in the vertical direction and a high Seebeck coefficient in the off-diagonal direction.

It is possible to tweak the design process so that one diagonal channel is obtained for larger $\Lambda$, cf. Fig. $4 d$ with Fig. 4e, but doing so will, according to our experience, not provide better design solution performances than already presented.

Apparently, the design solutions prefer only one simple diagonal channel of Material A, as more channels cause Material B to connect between $\Gamma^{N}$ and $\Gamma^{S}$ and hence causing a "thermal short circuit" which results in a high $f_{K}$ and hereby a low $f_{Z}$, please cf. Eq. (4).

As $f_{\alpha}$ has a squared weight in the objective function, the main feature of the design solutions is therefore to generate a high average electric potential along $\Gamma^{E}$ in the $\alpha$-load case. This attribute is maximized by the following two design features: (1) maximizing the heat flux normal to Material A in the diagonal channel and (2) choosing an adequate diagonal channel width to ensure that a sufficient amount of Material A is available for powering the Seebeck effect. 


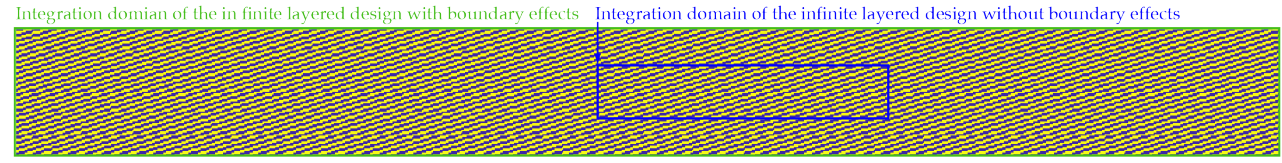

(a) Infinite layered design without boundary effects and infinite layered design with boundary effects.

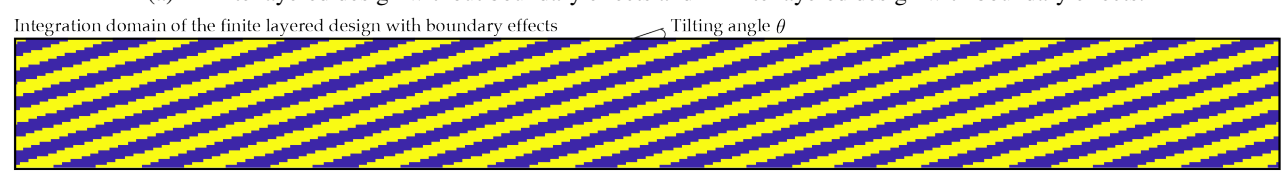

(b) Finite Layered design with boundary effects

Figure 3: The best performing design solutions obtained with the analytical optimization approach layout in Sakai et al. [3]. The design solutions are used to validate the topology optimization framework. The performances of the design solutions are compared in Fig. 2. The green, blue and black encircled squares indicate the integration domains in Eq. (5)

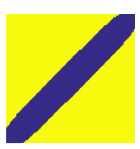

(a) $\Lambda=1$

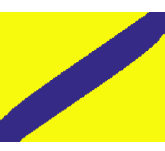

(b) $\Lambda=1.3$

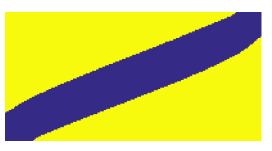

(c) $\Lambda=2$

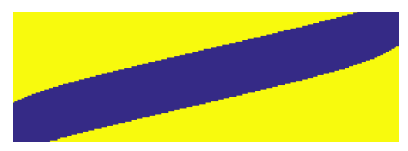

(d) $\Lambda=3$

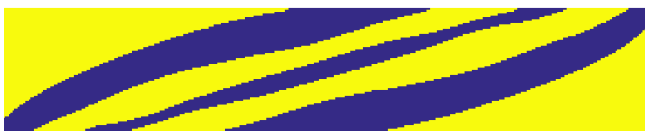

(e) $\Lambda=5$

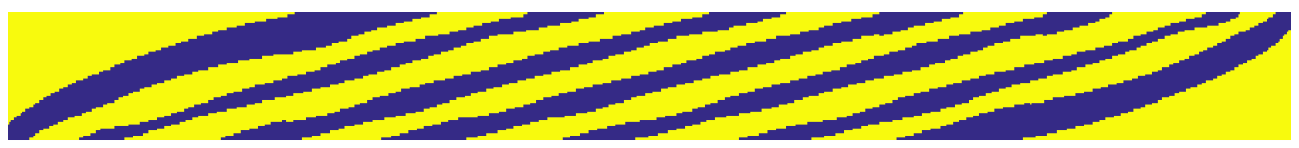

(f) $\Lambda=10$

Figure 4: Design solutions solved for $f_{Z}$ and various length-to-height ratios, $\Lambda$. The corresponding design solutions obtained with the analytical optimization approach in Sakai et al. [3] are plotted in Fig. 5. The performance of the design solutions are compared in Fig. 6, where it is seen that the best performing design solution is obtained for $\Lambda \approx 1.3$.

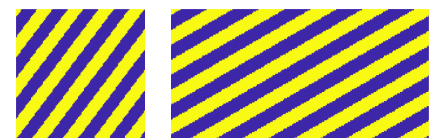

(a) $\Lambda=1$

(b) $\Lambda=2$

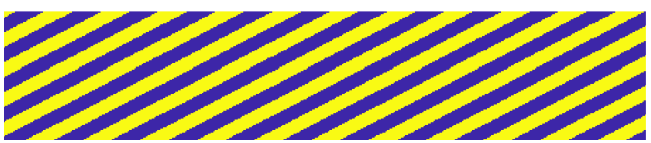

(c) $\Lambda=5$

Figure 5: The analytical designs solutions solved for $f_{Z}$ and various length-to-height ratios, $\Lambda$. The corresponding topology optimized design solution are plotted in Fig. 4. The performance of the design solutions are compared in Fig. 6. 


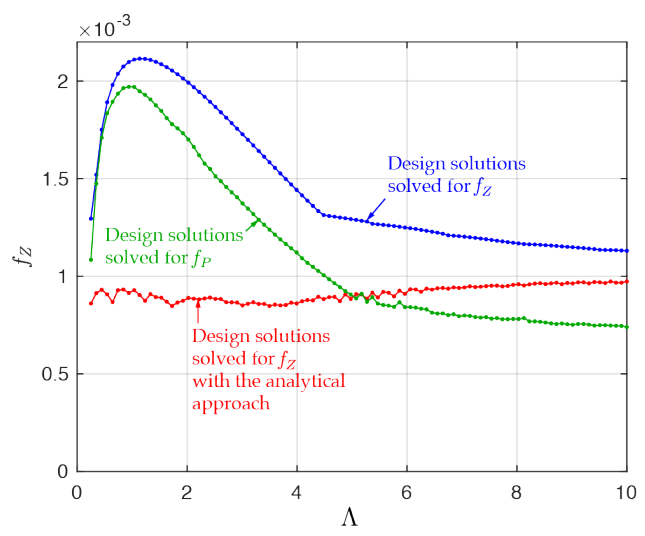

Figure 6: The relationship between the height-to-length ratio, $\Lambda$, and the off-diagonal figure-of-merit, $f_{Z}$, for the best performing design solutions obtained with the topology optimization approach and the solutions obtained with the topology optimization approach and the
analytical approach. The design solutions obtained with the topology analytical approach. The design solutions obtained with the topology
optimization framework and solved for $f_{Z}$ outperform the other deoptimization framework and solved for $f_{Z}$ outperform the other de
sign solutions. We therefore confidently conclude that the topology optimization approach is suited for optimizing the off-diagonal figureof-merit and that we may attribute significance to the features of the design solutions.

By comparing the design solutions in Fig. 4 and 5 , we observe that the relative differences between the design solutions of the two optimization approaches are decreased as $\Lambda$ is increased. As the relative differences between the design solutions are decreased, the relative performance differences are also decreased, please cf. Fig. 6

As a final remark, we call attention to that topology optimization is especially suited for off-diagonal problems, as trivial designs solutions, i.e. standalone Material A or $\mathrm{B}$, have $f_{\kappa} \neq 0, f_{\sigma} \neq 0$ and $f_{\alpha}=0$. The segmented configuration of off-diagonal thermoelectric generators is therefore necessary, because trivial design solutions don't generate any electric current.

\subsection{Electrical power output}

The goal of the second design problem is to optimize the electric power output, $f_{P}$, and again we use the design solutions obtained by the analytical optimization approach by Sakai and coworkers as benchmarks for the topology optimized designs solutions.

The design solutions solved with the analytical approach are obtained by a simple and computational expensive parameter study where both $\theta$ and $z^{E}$ are swept in 100 equally sized steps in the intervals $\theta \in[0 ; 90]$ [degrees] and $z^{E} \in[10 ; 15000][1 / \mathrm{S}]$, respectively.
The design solutions solved for $f_{P}$ and $\Lambda=$ $\{0.5,1,2,5,10\}$ have been plotted in Fig. 8. By com paring these design solutions with the solutions solved for $f_{Z}$ in Fig. 4, we notice that the choice of objective function has a considerable influence on the topology of the design solutions.

This difference is also reflected in the performances of the design solutions obtained with the different optimization approaches and objective functions. In Fig. 9 we have plotted the relationship between $\Lambda$ and $f_{P}$ for the different design solutions. The important point of the graph is the following: the design solutions solved for $f_{P}$ outperform all other design solutions, for which reason we confidently conclude that the choice of objective function is a critical parameter of the design problems and that the topology optimization methodology outperforms the analytical optimization approach.

Another interesting and important study is seen in Fig. $9 \mathrm{~b}$, where the electrical power output has been normalized with respect to $L_{x}$. This is done to demonstrate than an optimal choice for $\Lambda$ exists, if the designer's goal is to generate as much electrical power output as possible for a constrained length of the thermal hot and cold reservoirs. The maximal $f_{P} / L_{x}$ is obtained for $\Lambda \approx 1$ for reasons similar to those already discussed in Sec. 2.2.1.

\subsubsection{The features of the design solutions}

The main differences between the design solutions solved for $f_{Z}$ and $f_{P}$ can be characterized by two attributes: (1) the volume ratio of the materials and (2) the topology of the diagonal channels.

Design solutions solved for $f_{Z}$ generally have a larger amount of Material A in the diagonal than design solutions solved for $f_{P}$. This design feature is advantageous as an increased amount of thermoelectric active material powers the Seebeck effect and hereby increases the average electric potential difference on $\Gamma^{E}$.

The diagonal channels of Material A in the design solutions solved for $f_{P}$ tend to meet $\Gamma^{N}$ and $\Gamma^{S}$ at a perpendicular angle which is not the case for the design solutions solved for $f_{Z}$. This design feature results in reduced temperature gradients near $\Gamma^{N}$ and $\Gamma^{S}$, which increases the Peltier heat load and hereby contributes negatively to the electric current and the objective function. As the overall temperature variations are small for the design solutions solved for $f_{Z}$, this design feature is not advantageous for these problems.

The state field plots for the design solutions solved for $f_{P}$ and $\Lambda=1$ in Fig. $8 \mathrm{~b}$ have been plotted in Fig. 10 . The interplay between the design solution and the state fields are similar to those already discussed in Sec. 2.2.1. 


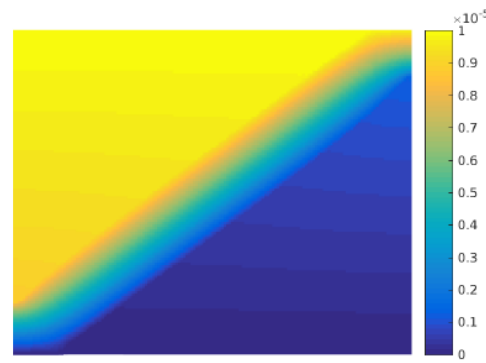

(a) Temperature $[\mathrm{K}]$

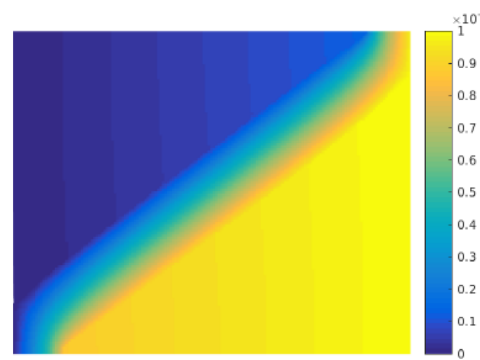

(c) Electric potential [V]

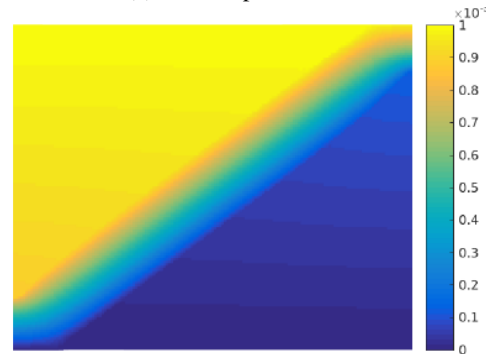

(e) Temperature $[\mathrm{K}$

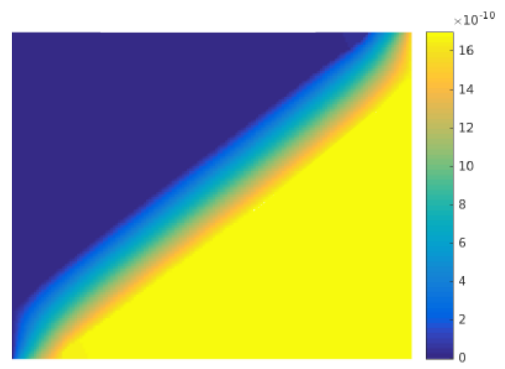

(b) Electric potential [V]

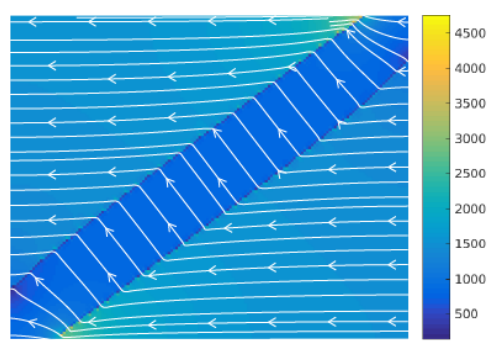

(d) Electric current density $\left[\mathrm{A} / \mathrm{m}^{2}\right]$

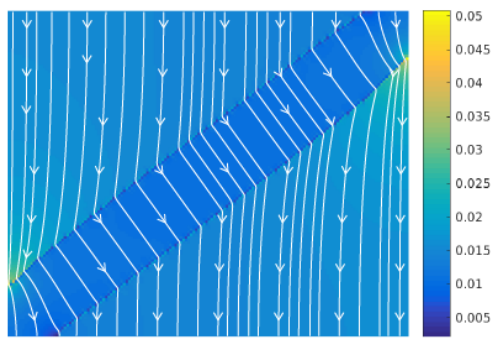

(f) Heat flux $\left[\mathrm{W} / \mathrm{m}^{2}\right]$

Figure 7: The relevant state field plots of the $\alpha$-load case, (a) and(b), the $\sigma$-load case, (c) and (d), and the $\alpha$-load case, (e) and (f), for the design solution solved for $f_{\mathrm{Z}}$ and $\Lambda=1.3$ in Fig. 4b. With reference to the large gradients of the electric potential field in the diagonal channel, the surrounding metal is working as a thermal and electric conductor and the diagonal channel is powering Seebeck effect and hereby the thermoelectric surrounding metal 


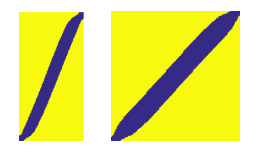

(a)

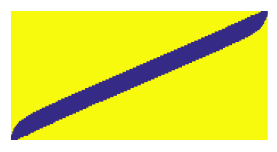

(c) $\Lambda=2$

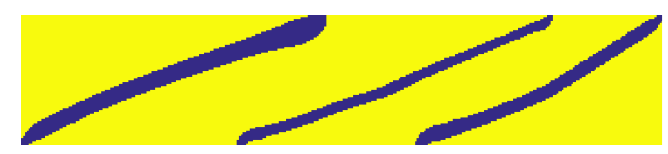

(d) $\Lambda=5$

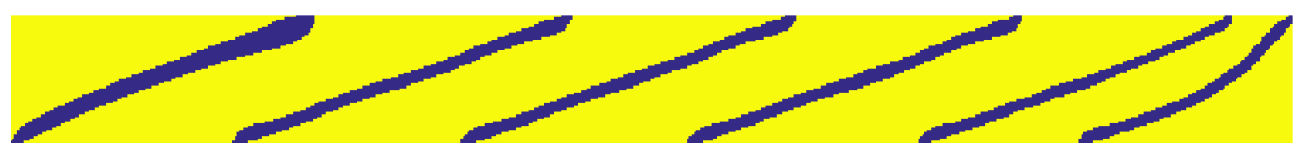

(e) $\Lambda=10$

Figure 8: Design solutions solved for $f_{P}$ and various length-to-height ratios, $\Lambda$. By comparing the design solutions solved for $f_{P}$ in present figure, the design solutions solved for $f_{Z}$ in Fig. 4 and the performances of the design solutions in Fig. 9 , we call attention to (A) the considerably different design solutions solved for different objective functions and (B) the superior performance of the design solutions solved for $f_{P}$. We hereby conclude that the choice of objective function is a critical design parameter and should be taking into consideration in design problems of thermoelectric generators.

\subsubsection{The impedance of the outer resistive load}

The impedance of the outer resistive load is part of the design problem, and to demonstrate the importance of the model parameter, we have plotted the design solutions solved for $f_{P}, z^{E}=\{150,8000,15000\}$ and $\Lambda=2$ in Fig. 11. The design solutions are considerably different, which is also reflected in the corresponding relationships between $f_{P}$ and $z^{E}$ for the three design solutions plotted in Fig. 12.

The relationships between $f_{P}$ and $z^{E}$ show that a design solution solved and evaluated for a specific $z^{E}$ has superior performance compared to design solutions solved and evaluated for another $z^{E}$. The impedance of the outer resistive load is therefore a critical model parameter and should be taken into account for design problem involving off-diagonal thermoelectric generators.

\subsection{Diagonal and off-diagonal problems}

In previous sections we have demonstrated that topology optimization can be used to outperform other optimization approaches in the literature. However, we have not discussed the performance difference between off-diagonal and diagonal thermoelectric generators. Diagonal thermoelectic generators convert a vertical heat flux into a vertical electric current, and this configuration is the most standard and applied configuration of thermoelectic generators.

Tab. 5 shows that diagonal thermoelectric generators outperform off-diagonal thermoelectric generators for both $f_{Z}$ and $f_{P}$, however the performance loss incurred by using the off-diagonal thermoelectric generators may be acceptable when taking into account that the electrodes on $\Gamma^{E}$ and $\Gamma^{W}$ are not required to be in contact with the heat source. By decoupling the electrodes and the heat source, the probability of damaging the electrodes with thermo-mechanical stresses and wear during operation is decreased and this may be game changing in applications where the temperature difference between the hot and the cold reservoirs are changing considerable during operation.

\subsection{Boundary conditions}

We claimed in Sec. 1.3 that adequate modeling for the thermal heat transfer between the hot and cold reservoirs and the thermoelectric generator is constituted by Newton's law of cooling. The plot in Fig. 13 is key to support this claim.

With basis in a diagonal thermoelectric generator, the plot shows the relationship between the height of the design domain, $L_{y}$, and the electric power output, $f_{P}$, for various heat transfer rates. For finite heat transfer rates there exist an optimum between the electrical power output and the height of the design domain. However, as the heat transfer rate goes to infinity, the optimal height of the design domain goes to zero, and the electric power output goes to infinity. This singularity is indeed nonintuitive and non-physical and it is therefore concluded that finite heat transfer rates between the hot and cold reservoirs and the thermoelectric generator are required for carrying out adequate physical modeling for thermoelectric generators. 
Table 5: A comparison between the figure-of-merit, $f_{Z}$, electrical power output, $f_{P}$, and the normalized electrical power output, $f_{P} / L_{x}$ for design solutions of standalone materials in diagonal and off-diagonal configurations. The topology optimized design solution solved in the diagonal configuration (not shown in the paper) outperform the design solutions solved in the off-diagonal configurations. However, thermoelectric generator in off-diagonal configurations may be less prone the thermo-mechanical stresses and wear.

\begin{tabular}{llll}
\hline Description & Type & $f_{P} / L_{x}$ & $f_{Z}$ \\
\hline Standalone Material A & Diagonal & 1444.18 & $3.341 \cdot 10^{-3}$ \\
Standalone Material B & Diagonal & 85.17 & $0.046 \cdot 10^{-3}$ \\
\hline Design solution & Diagonal & 4378.62 & $3.302 \cdot 10^{-3}$ \\
Design solution in Fig. 8b & Off-diagonal & 3896.19 & $2.110 \cdot 10^{-3}$ \\
\hline
\end{tabular}

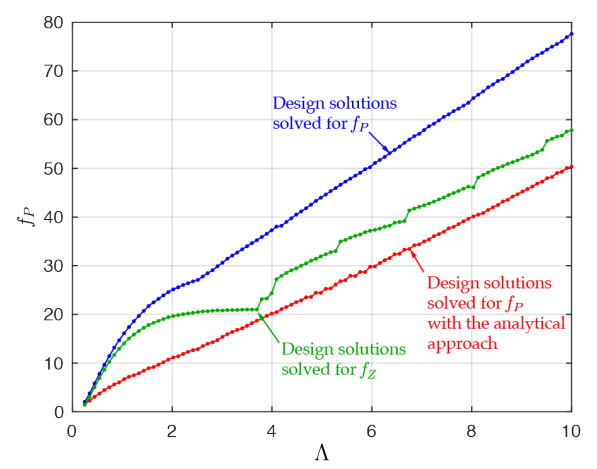

(a) Electrical power output as function the length-to-height ratio

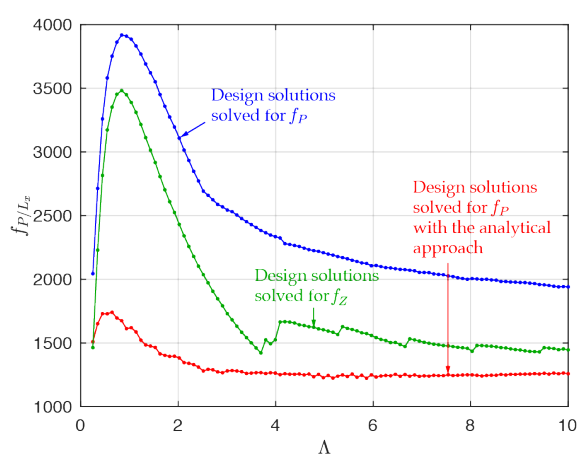

(b) Normalized electrical power output as function of the lengthto-height ratio

Figure 9: The relationships between the length-to-height ratio, $\Lambda$, the electrical power output, $f_{P}$, and the normalized electrical power output, $f_{P} / L_{x}$ for the design solutions solved for $f_{P}$ and $f_{Z}$. The design put, $f_{P} / L_{x}$ for the design solutions solved for $f_{P}$ and $f_{Z}$. The design
solutions solved for $f_{P}$ with topology optimization outperform design solutions solved for other objectives and optimization approaches, for which reason we conclude that the topology optimization approach is suited for optimizing the off-diagonal electric power output of thermoelectric generators. 


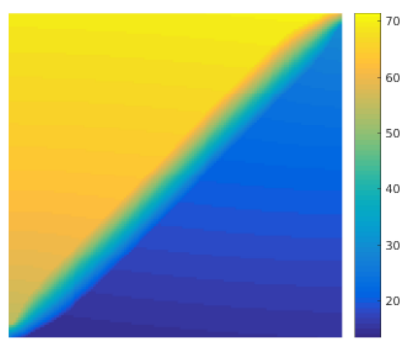

(a) Temperature $[\mathrm{K}]$

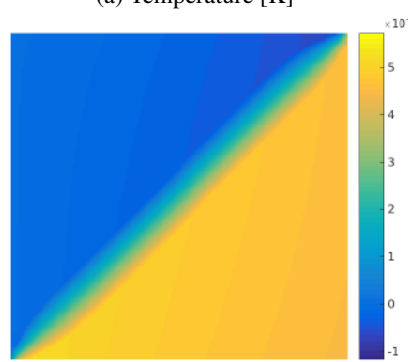

(b) Electric potential [V]

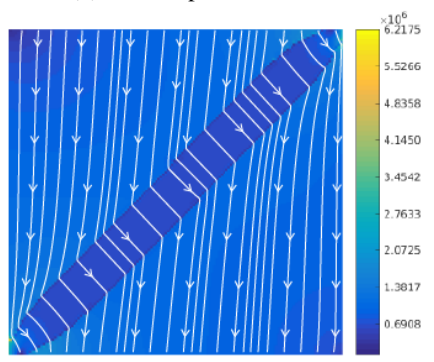

(c) Heat flux $\left[\mathrm{W} / \mathrm{m}^{2}\right]$

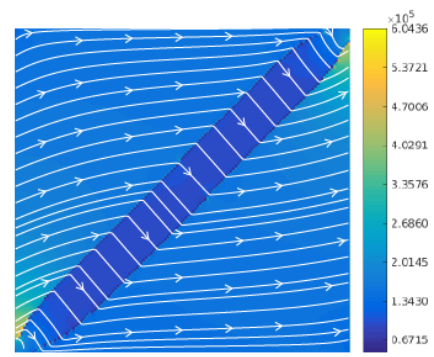

(d) Electric current density $\left[\mathrm{A} / \mathrm{m}^{2}\right]$

Figure 10: The state field plots for the design solution solved for electrical power output, $f_{P}$, and length-to-height ratio, $\Lambda=1$, in Fig. $8 \mathrm{~b}$. The design features of the design solution are equivalent to what already discussed in the caption in Fig. 7 and in Sec. 2.3.1.
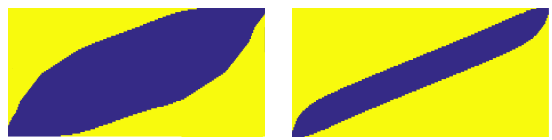

(a) $z_{\text {imp }}=150$

(b) $z_{\text {imp }}=8000$

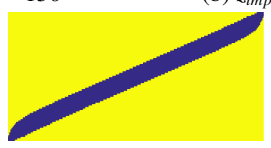

(c) $z_{\text {imp }}=15000$

Figure 11: Design solutions solved for electrical power output, $f_{P}$, and different electrical impedances of the outer resistive load, $z^{E}$. The performance of the design solutions are plotted in Fig. 12. As the performance and topology of the design solutions are dependent on $z^{E}$, we conclude that $z^{E}$ is an important model parameter.

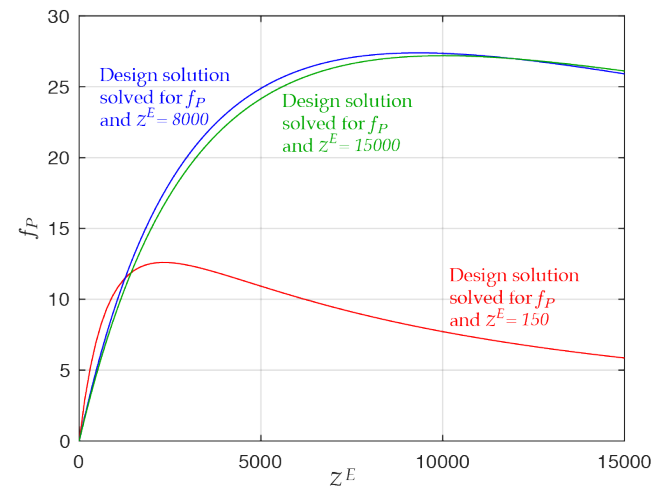

Figure 12: The relationship between the electrical power output, $f_{P}$, and the electrical resistance in the outer load, $z^{E}$, for the design solutions plotted in Fig. 11. Design solutions solved for one $z^{E}$ outperform design solutions solved for other $z^{E}$ for which reason we conclude that we can attribute importance to the features to the design solutions. 


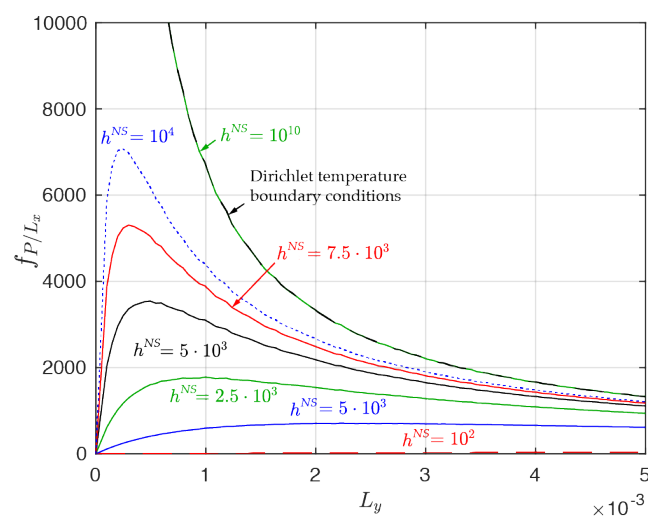

Figure 13: The relationship between the normalized electrical power output, $f_{P} / L_{x}$, the length of the design domain, $L_{y}$, and various convection coefficients, $h^{N S}$, for trivial design solutions consisting of standalon Material A. As the convection coefficients are increased, the electric power output are increased and the optimal design domain length is decreased. Dirichlet type boundary conditions are equivalent to $h^{N S} \rightarrow \infty$, which due to the singularity between $f_{P} / L_{x}$ and $L_{y}$ is concluded to be non-physical.

\section{Discussion}

All results presented in the present paper are supported by validation studies, benchmark examples and cross-checks, for which reason we confidently conclude that the topology optimization approach is a promising methodology for optimizing the performance of segmented off-diagonal thermoelectric generators. However, there are three issues that need to be discussed in relation to the presented results:

1. The neglection of parasitic losses

2. The manufacturability of the design solutions

3 . The assumption of temperature independent material parameters

These topics are discussed in the following.

\subsection{Parasitic losses}

The thermal and electric parasitic losses in the transitions between the material phases are neglected in the underlying finite element model. The work presented is hence purely theoretical and the design solutions have not been tested experimentally.

The losses should indeed be included in detailed computations, however we argue that the methodology and design solutions are relevant despite neglecting the losses.
Our main argument can be divided in three subarguments:

(A) [3] presented an analytical optimization approach which was used to optimize the figure-of-merit and the electric power output. This study is especially interesting because the design solutions were manufactured and tested experimentally. Even though the design solutions consisted of a high number of transitions between material phases, Sakai and coworkers found good agreement between the experimental measurements and the analytical predictions. Since our designs have less interface regions, we therefore argue that it is reasonable to assume that the thermal and electrical parasitic losses are smaller for the design solutions presented in this study than the design solutions tested in by Sakai and coworkers.

(B) Off-diagonal thermoelectric generators are required to be segmented with two or more different materials to produce thermoelectric energy, please confer the discussion in Sec. 2.2.1. As segmentation is necessary to ensure the functionality of off-diagonal thermoelectric generators, designers are therefore forced to accept parasitic losses which make the topology optimization approach, used in the present study, suitable.

(C) With performance improvement beyond $\approx 200 \%$ compared to analytical design solutions, we argue that these performance improvements are so large that they may dominate the parasitic losses.

\subsection{Manufacturability of the design solutions}

The manufacturability of the design solutions can be assessed with basis in three characteristica of the design solutions: (A) the topology of the design features (B) the number of transitions between the material phases and (C) the minimum length scale.

(A) The analyical design solutions tested and manufactured by [3] were quite similar to the design solutions presented in the present study as the design solutions obtained with the numerical and the analytical approach both consisted of simple geometrical structures such as triangles and parallelograms, see e.g Figs. $4 \mathrm{~b}$ and $8 \mathrm{~b}$.

(B) With respect to the number of transitions between the material phases, the analytical design solutions consisted of a considerably amount of diagonal channels compared to the design solutions with only one diagonal channel seen in the present study. The transitions between the material phases are a critical part in relation to the manufacturability, as poor connections between the material phases result in poor transfer of thermal and electrical energy and hereby high parasitic losses. However, with reference to the good agreements between the analytical predictions and experiments in Sakai and coworkers, it seems that they overcame this challenge. 
The design solutions presented in the present paper are solved with a requirement on the minimum length scale and the design solutions does therefore not contain design features which are smaller than a prescribed value. This approach provides a road to tailor the design solutions such that they are suited for additive manufacturing techniques as the minimum length-scales of the design features are matched to the available printing resolution.

With reference to the study by Sakai and coworkers, we point out that the topology optimized design solutions are simpler or equally simple in topology, have smaller amount of transitions between the material phases, and the same minimum length scale, for which reason we assess that the design solutions presented in the present paper are less or equally complicated to manufacture compared to the design solutions presented in the work of Sakai and coworkers.

\subsection{Temperature independent materials}

The design problems were solved for temperature independent material parameters, which indeed is an inadequate assumption for many physical materials. We decided to limit the design problems to temperature independent materials to ensure a frame of reference with respect to the design solutions presented in Sakai et al. [3]. The topology optimization methodology can straight forwardly be extended such that temperature dependent material parameters in taking into consideration. Implementation details are provided in Lundgaard and Sigmund [6].

\section{Conclusion}

We have used a density-based topology optimization methodology to optimize the figure-of-merit and the electric power output for off-diagonal thermoelectric generators. The objectives are optimized by spatially distributing two different thermoelectric materials in a two dimensional design space.

With basis in a fully coupled thermoelectric model with temperature independent materials, we use the framework to identify and discuss several important design model parameters. The most important findings have been listed in the following:

1. With reference to the excellent agreements between the analytic predictions in Yang et al. [14], Sakai et al. [3], the experimental validations in [3] and the topology optimization framework, we confidently conclude that we can rely on the underlying mathematical model of the framework.
2. The topology optimized design solutions outperform the analytical design solutions by $233 \%$ for design problems solved for figure-of-merit and by $229 \%$ for design problems solved for electric power output. The topology optimization approach is therefore concluded to be excellently suited for optimizing off-diagonal thermoelectric generators.

3. Design solutions solved for figure-of-merit do not necessarily provide large electrical power outputs and design solutions solved for electric power output do not necessarily provide large figure-of-merits. The choice of objective function is therefore concluded to be a critical design parameter and designers should therefore carefully consider the interplay between the end-goal application of the devices and the optimization problem.

4. By solving three different realization of the design problems in a min/max formulation, it is possible to control the length-scales of the design solutions, such that they are supported by the resolution of the manufacturing techniques available.

5. To ensure adequate physical modeling, the heat transfer rates between the cold reservoir, the hot reservoir and the thermoelectric generator are finite to ensure physical realistic modeling. Surfaces with Dirichlet type temperature boundary conditions are subject to infinite heat transfer rates, for which reason these type of boundary condition may result in non-physical physical modeling and therefore useless design solutions.

6. With basis in the state fields of the design solutions, we conclude that the spatial distribution and the volume ratio between the materials are the primary design features for maximizing the figure-of-merit and the electric power output.

7. By analyzing crosscheck studies, we identify the length of the thermoelectric generator and the impedance in the outer electrical load as critical design parameters for maximizing the figure-of-merit and the electric power output.

8. Diagonal thermoelectric generators outperform offdiagonal thermoelectric generators for both electric power output and figure-of-merit, however offdiagonal thermoelectric generators may be less prone to wear and/or thermo-mechanical stresses.

The methodology can easily be extended to incorporate temperature dependent materials, multiple materials phases, other objective functions and different boundary conditions. However, for now, the study serves as a demonstration that topology optimization is a suitable 
method for optimizing off-diagonal thermoelectric generators.

\section{Acknowledgements}

The authors acknowledge the financial support received from the TopTen project sponsored by the Danish Council for Independent Research (DFF-4005-00320).

\section{Bibliography}

[1] David Michael Rowe. Thermoelectrics handbook: macro to nano. CRC press, 2005.

[2] Dario Narducci. Do we really need high thermoelectric figures of merit? A critical appraisal to the power conversion efficiency of merit? A critical appraisal to the power conversion efficiency of
thermoelectric materials. Applied Physics Letters, 99(10):10-13, thermoelectric materials. Applied Physics Letters,

[3] Akihiro Sakai, Tsutomu Kanno, Kouhei Takahashi, Hiromasa Tamaki, Hideo Kusada, Yuka Yamada, and Hiroya Abe. Breaking the trade-off between thermal and electrical conductivities in the thermoelectric material of an artificially tilted multilayer. Scientific reports, 4:6089, 2014.

[4] Martin Philip Bendsøe and Noboru Kikuchi. Generating optimal topologies in structural design using a homogenization method. Computer methods in applied mechanics and engineering, 71(2): 197-224, 1988

[5] Martin Bendsøe and Ole Sigmund. Topology Optimization Theory, Methods and Applications. Springer, 2003.

[6] Christian Lundgaard and Ole Sigmund. A density-based topology optimization methodology for thermoelectric energy conversion problems. Structural and Multidisciplinary Optimization, pages 1-16, 2018.

[7] Elena E Antonova and David C Looman. Finite Elements for Thermoelectric Device Analysis in ANSYS. International Conference on Thermoelectrics, pages 1-4, aug 2000.

[8] Robert D. Cook, David S. Malkus, Michael E. Plesha, and Robert J. Witt. Concepts and applications of finite element analysis. John Wiley \& Sons, 4th edition, 2007.

[9] Panagiotis Michaleris, Daniel A Tortorelli, and Creto A Vidal. Tangent operators and design sensitivity formulations for transient non-linear coupled problems with applications to elastoplasticity. International Journal for Numerical Methods in Engineering, 37(14):2471-2499, 1994.

[10] Krister Svanberg. The method of moving asymptotesa new method for structural optimization. International journal for numerical methods in engineering, 24(2):359-373, 1987.

[11] York Christian Gerstenmaier and Gerhard Wachutka. Unified theory for inhomogeneous thermoelectric generators and coolers including multistage devices. Physical Review E - Statistical, Nonlinear, and Soft Matter Physics, 86:1-10, 2012. ISSN 15393755. doi: 10.1103/PhysRevE.86.056703.

[12] TS Ursell and GN Snyder. Compatibility of segmented thermoelectric generators. In Thermoelectrics, 2002. Proceedings ICT'02. Twenty-First International Conference on, pages 412417. IEEE, 2002.

[13] Alexander Heghmanns and Michael Beitelschmidt. Parameter optimization of thermoelectric modules using a genetic algorithm. Applied Energy, 155:447-454, 2015.

[14] Y Yang, S H Xie, F Y Ma, and C H Lei. On the effective thermoelectric properties of layered heterogeneous medium. Journal of Applied Physics, 111(1):3510, January 2012.
[15] Y Yang, F Y Ma, C H Lei, Y Y Liu, and J Y Li. Nonlinear asymptotic homogenization and the effective behavior of layered thermoelectric composites. Journal of the Mechanics and Physics of Solids, 61(8):1768-1783, 2013. doi: 10.1016/j.jmps.2013.03.006.

[16] Yang Yang, Chihou Lei, Cun Fa Gao, and Jiangyu Li. Asymptotic homogenization of three-dimensional thermoelectric composites. Journal of the Mechanics and Physics of Solids, 76:98-126, 2015. doi: 10.1016/j.jmps.2014.12.006.

[17] SP Yushanov, LT Gritter, JS Crompton, and KC Koppenhoefer. Multiphysics analysis of thermoelectric phenomena. In Seventh Annual Conference on Multiphysics Modeling and Simulation. Proceedings of the 2011 COMSOL Conference, Boston, USA, 2011.

[18] Y Yang, F Y Ma, C H Lei, and Y Y Liu, Is thermoelectric conversion efficiency of a composite bounded by its constituents? Applied Physics Letters, 102(5):53905, January 2013.

[19] Fengwen Wang, Boyan Stefanov Lazarov, and Ole Sigmund. On projection methods, convergence and robust formulations in topology optimization. Structural and Multidisciplinary Optimization, 43(6):767-784, dec 2011.

[20] James K Guest and Jean H Prévost. Topology optimization of creeping fluid flows using a Darcy-Stokes finite element. International Journal for Numerical Methods in Engineering, 66(3): 461-484, 2006.

[21] Ole Sigmund. Morphology-based black and white filters for topology optimization. Structural and Multidisciplinary Optimization, 33(4-5):401-424, jan 2007. 